\title{
Schwangerschaft, Geburt und Wochenbett bei Frauen mit Querschnittlähmung
}

Ute Lange, Markus Schmidt, Ines Kurze

Die Betreuung von schwangeren Frauen mit Querschnittlähmung erfordert die fachübergreifende Zusammenarbeit von Ärzten und Hebammen. Darin liegt auch eine große Chance. Denn trotz bestehender Risiken, sollte es immer darum gehen, den Frauen ein positives Erleben von Schwangerschaft, Geburt und Wochenbett zu ermöglichen.

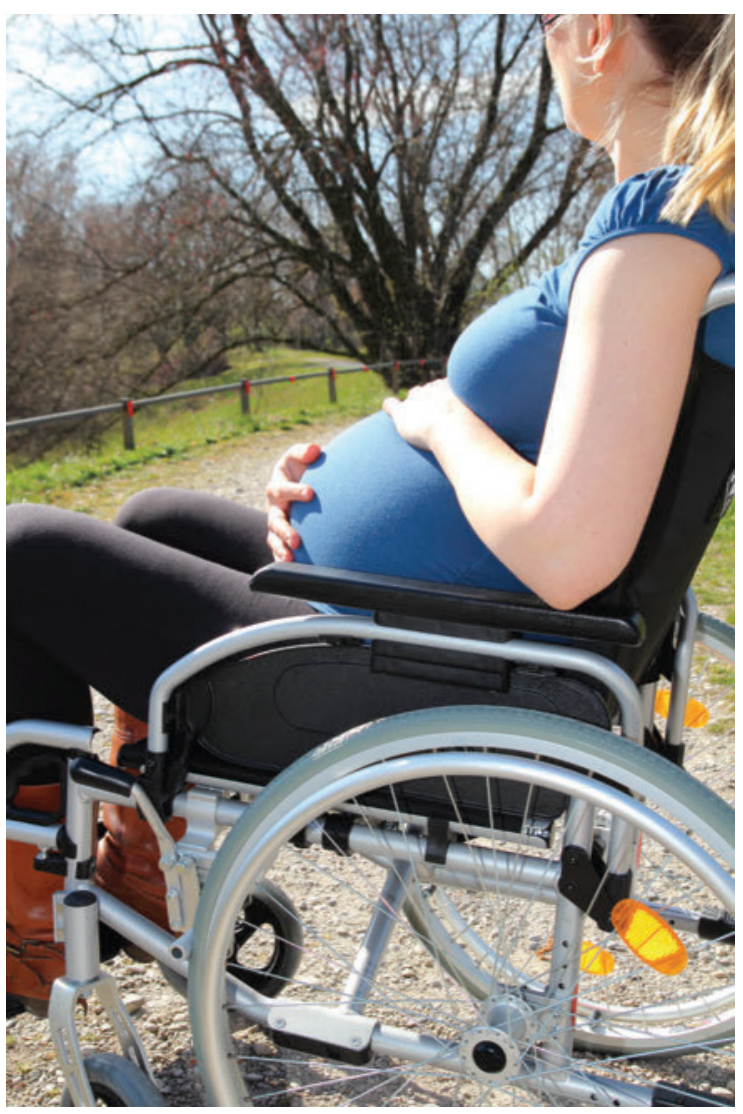

Abb. 1 Der Wunsch, eigene Kinder zu haben, ist für Frauen mit Querschnittlämung genauso selbstverständlich wie für Frauen ohne Handicap. Was für die Betreuung in Schwangerschaft, Geburt und Wochenbett wichtig ist, fassen die Mitautoren der Leitliniengruppe in diesem Artikel zusammen. (Foto: RioPatuca Images - stock.adobe. com, Symbolbild)

\section{Hintergrund}

Die Lebenserwartung und die Lebensqualität von rückenmarkgeschädigten Menschen konnte durch die verbesserte medizinische Versorgung in den letzten Jahrzehnten deutlich erhöht bzw. verbessert werden. Familienplanung und Kinderwunsch gehören daher heute ganz selbstverständlich zum Lebensentwurf von Frauen mit einer Querschnittlähmung (QSL). Internationale Studien zeigen Schwangerschaftsraten von 14-18\%, wobei sich die meisten Studien nur auf kleine Stichproben beziehen [22][15]. Grundlegende epidemiologische Informationen über Schwangerschaftsraten bei querschnittgelähmten Frauen sind für Deutschland bisher nicht verfügbar. Bekannt ist, dass neben sozialen Faktoren wie Familienstand, Schulbildung und Beruf insbesondere der Zeitpunkt des Lähmungseintritts Einfluss auf eine Mutterschaft mit QSL hat.

Aufgrund der allgemein begrenzten Datenlage und bei häufig nur geringem persönlichen Erfahrungsschatz herrscht insgesamt eine große Unsicherheit bei behandelnden Fachärzten sowie Hebammen in der geburtshilflichen Versorgung betroffener Frauen. Die kürzlich publizierte S2k-Leitlinie mit dem Titel „Schwangerschaft, Geburt und Wochenbett bei Frauen mit Querschnittlähmung“ versucht, eine Hilfestellung bei der Etablierung interdisziplinärer Standards zu geben [2]. Der folgende Beitrag fasst einige wichtige praxisrelevante Aspekte dieser Leitlinie zusammen und ergänzt sie um Aspekte, die insbesondere für die Hebammenversorgung bedeutsam sind.

\section{Präkonzeptionelle Beratung}

Einer präkonzeptionellen Beratung betroffener Frauen kommt eine große Bedeutung zu. Sie hat zum Ziel, 
den Gesundheitszustand vor einer Schwangerschaft zu optimieren und damit eine Primärprävention sowohl im medizinischen als auch im sozialen Bereich zu erzielen [37][19]. Diese präkonzeptionelle Planung sollte durch einen Gynäkologen/Geburtshelfer in enger Abstimmung mit dem behandelnden Paraplegiologen bzw. Neuro-Urologen koordiniert werden [19][8]. Die Beratung beinhaltet u.a. das Harnblasenmanagement, Darmmanagement und den Umgang mit möglichen Implantaten.

In einem präkonzeptionellen Gespräch sollten die neurologische, orthopädische und urologische Stabilität der Frau evaluiert, die bestehende Medikation überprüft, umgestellt und ggf. pausiert werden. Diese eventuell erforderliche Umstellung einer bestehenden Medikation sollte in jedem Fall in enger Absprache mit dem betreuenden Paraplegiologen Neuro-Urologen erfolgen. Es kann weiterhin eine unabhängige Beratung durch das Pharmakovigilanz- und Beratungszentrum für Embryonaltoxikologie (Embryotox) der Charité - Universitätsmedizin Berlin geleistet werden.

Insbesondere bei Frauen mit Spina bifida oder Neuralrohrdefekten sollte eine erhöhte Folsäure-Gabe von $4 \mathrm{mg}$ pro Tag, möglichst 2-3 Monate präkonzeptionell erfolgen [13]. Bei allen anderen querschnittgelähmten Frauen sollte die zumeist übliche Dosis von $0,4 \mathrm{mg}$ pro Tag ergänzt werden [16].

\section{Betreuung während der Schwangerschaft}

\author{
Merke \\ Eine Schwangerschaft bei Frauen mit QSL ist gemäß \\ der Mutterschaftsrichtlinie grundsätzlich als Risiko- \\ schwangerschaft einzustufen. Dies bedingt einerseits, \\ dass Zusatzuntersuchungen durchgeführt und Unter- \\ suchungsintervalle verkürzt werden. Andererseits \\ besteht die Notwendigkeit einer interdisziplinären \\ Betreuung während der gesamten peripartalen Phase.
}

Eine frühzeitige vorgeburtliche Vorstellung in der Geburtsklinik sowie in einer anästhesiologischen Sprechstunde ist zwingend erforderlich. Bei jeder zweiten Schwangerschaft von Frauen mit einer Querschnittlähmung kommt es zu einer vorgeburtlichen Hospitalisierung wegen Harnwegsinfektionen und Harninkontinenz, Hypertonie, Pneumonie, Präeklampsie sowie vorzeitigen Wehen und Tachykardien [9].

Schwangere mit QSL sollten möglichst früh mit einer Hebamme Kontakt aufnehmen. In der Hebammenbetreuung steht die Begleitung der Frau und Familie in der Phase der Familienbildung im Vordergrund. Der Alltag von Menschen mit QSL ist meist mit einem höheren organisatorischen Aufwand verbunden, was bei der Beratung und bei den Empfehlungen an die Frauen und Familien berücksichtigt werden muss. Zudem sind häufig Dritte in verstärktem Maße in die Alltagsgestaltung eingebunden. Auch diese gilt es wertzuschätzen und gegebenenfalls in die Betreuungskonzepte zu integrieren [10].

\section{Harnblasen- und Darmmanagement}

Merke

Die aufgrund der Querschnittlähmung bestehende neurogene Dysfunktion des unteren Harntraktes und neurogene Darmfunktionsstörung kann während der Schwangerschaft verändert sein. Daher muss das Management entsprechend angepasst werden [3].

Urologische Probleme wie Harnwegsinfektionen oder Inkontinenz stellen die häufigste Komplikation bei Schwangeren mit Querschnittlähmung dar [34][38][7] [23]. Es werden deutlich höhere Raten an Harnwegsinfekten bei Frauen mit Querschnittlähmung (45,5\%) gegenüber den Schwangeren ohne Querschnittlähmung (8,2\%) beschrieben [24]. Vierwöchentliche Urinkontrollen inklusive der Urinkultur via Katheterurin sollten daher ebenso zur Vorsorge gehören wie regelmäßige Sonografien des Harntraktes und ggf. laborchemische Untersuchungen. Prinzipiell wird bei Bedarf eine länger andauernde (7-10 Tage) und höher dosierte antibiotische Therapie als üblich durchgeführt. Dies gilt auch beim Auftreten einer asymptomatischen Bakteriurie mit entsprechender Leukozyturie. Eine antibiotische Dauerprophylaxe bei Schwangeren mit QSL sollte außer nach einer Pyelonephritis bei fehlender Evidenz und bei der Gefahr der Verschlechterung der Resistenzlage nicht erfolgen. Als Mittel der Wahl gelten Beta-Laktam-Antibiotika sowie Cephalosporine. Einer Antibiotikatherapie sollte stets eine Urinkultur mit Antibiogramm vorausgehen [34][38][7][23].

In der Schwangerschaft ist mit einer erhöhten Rate an Obstipationen zu rechnen [32][30]. Um die Darmpassage zu unterstützen, sollten ballaststoffreiche Kost und ggf. zusätzlich Lactulose als Weichmacher eingesetzt werden. Die Hebamme kann mit der Schwangeren bei Bedarf einen auf ihren Lebensstil ausgerichteten Ernährungsplan aufstellen. Bei hartnäckiger Obstipation kann kurzfristig Natriumpicosulfat angewandt werden. Die digitale Stimulation und ggf. digitale Ausräumung sollte primär zur Entleerung der Rektum-Ampulle eingesetzt werden. Zur Unterstützung der rektalen Entleerung sind $\mathrm{CO}_{2}$-haltige Zäpfchen unbedenklich.

\section{Umgang mit Implantaten}

Frauen mit QSL können mit Implantaten zur sakralen Neuromodulation, zur sakralen Vorderwurzelstimulation, aber auch mit künstlichen Blasenschließmuskeln sowie Medikamentenpumpen und ventrikulo-peritonealen bzw. ventrikulo-atrialen Shunt-Systemen versorgt sein. Die 
Studienlage zum Umgang mit diesen Implantaten in der Schwangerschaft ist unzureichend [35][25][31][40]. Dennoch empfiehlt die Leitliniengruppe bei querschnittgelähmten Schwangeren mit sakraler Neuromodulation die Beibehaltung der Aktivierung während der Schwangerschaft. Erfolgt eine sakrale Vorderwurzelstimulation zur Entleerung der Harnblase, so kann diese in der Schwangerschaft ebenfalls fortgeführt werden. Ein urethraler Sphinkterersatz stellt in Ermangelung aussagekräftiger Literaturdaten und der Gefahr einer potenziellen Schädigung von Urethra, Blase und des eingesetzten Sphinkters eine Indikation zur primären Sectio dar. Es existieren weiterhin nur wenige veröffentlichte Fälle, welche den Schwangerschaftsverlauf mit implantierter intrathekaler Baclofen-Pumpe beschreiben. Die Komplikationsrate wird insgesamt als niedrig beschrieben. Eine intrathekale Baclofen-Applikation scheint für das Kind sicher zu sein. Bei Frauen mit implantierter Medikamentenpumpe sollte großzügig eine Sectioindikation gestellt werden.

\section{Thromboseprophylaxe}

Aufgrund hormoneller Veränderungen, veränderter Druckverhältnisse im Bauchraum und einem verlangsamten Blutfluss, besteht in der Schwangerschaft bei Frauen mit QSL - wie auch bei nicht gelähmten Schwangeren insbesondere im Becken- und Beinvenenbereich ein erhöhtes Thromboserisiko. Eine generelle Indikation zur Thromboseprophylaxe bei Rollstuhlfahrerinnen mit QSL ohne zusätzliche Erkrankung besteht nicht. Die Prävention venöser Thromboembolien basiert auf der sorgfältigen Erfassung bereits bestehender und neu aufgetretener Risikofaktoren.

Aufgrund einer zunehmenden Immobilität im letzten Drittel der Schwangerschaft empfiehlt die Leitliniengruppe ab der 28. SSW bis 6 Wochen postpartal eine generelle medikamentöse Thromboseprophylaxe. Bei zusätzlichen Risikofaktoren sollte eine Prophylaxe in der gesamten Zeit der Schwangerschaft und im Wochenbett bis 6 Wochen postpartal durchgeführt werden. Niedermolekulare Heparine gelten als Medikamente der Wahl. Weiterhin sollten regelmäßiges Hochlagern der Beine, kalte Beinduschen, vermehrte passive oder aktive Bewegung sowie das Tragen von Stützstrümpfen empfohlen werden [20].

\section{Therapie von Harnwegsinfekten}

Schwangere mit QSL haben im Vergleich zu anderen Frauen eine Verschlechterung der Blasenfunktion und in deren Folge assoziierte Schwangerschaftskomplikationen. So haben Harnwegsinfekte einen signifikanten Einfluss auf die Frühgeburtenrate [33]. Entsprechend kommt es bei 33-60\% der Frauen mit QSL zu einer vorzeitigen Geburt [29][12]. Aus diesem Grund muss das Blasenmanagement durch Verkürzung der Katheterisierungsintervalle sowie in Einzelfällen der Anlage einer Harndauerableitung angepasst werden. Ein bestehender Harnwegsinfekt sollte konsequent auch bei asymptomatischer Bakteriurie therapiert werden.

\section{Frühgeburtsrisiko}

Da bei Menschen mit QSL aufgrund der eingeschränkten Sensibilität und Schmerzempfindung sowie möglichen Temperaturregulationsstörungen die klassischen Symptome wie Flankenschmerz und/ oder Fieber fehlen können, ist neben der klinischen Symptomatik der paraklinische Status (CRP, Leukozytose) wegweisend. Die Problematik einer drohenden Frühgeburt wird dadurch weiter verstärkt, dass die Frauen die vorzeitige Wehentätigkeit häufig nicht spüren [12].

\section{Autonome Dysreflexie}

Eine Autonome Dysreflexie (AD) wird typischerweise durch eine Stimulation ausgelöst, wie sie bei Wehen, Harnverhalten, Überdehnung des Darms sowie einer vaginalen sowie rektalen Manipulation vorkommt. Für Fachpersonen wie Gynäkologen und Hebammen ist es wichtig, zu wissen, dass Schmerzreize im gelähmten Bereich, auch wenn sie nicht bewusst wahrgenommen werden, der Auslöser für eine AD sein können [27]. Eine $A D$ tritt typischerweise bei Schwangeren mit einer Lähmungshöhe oberhalb des thorakalen Rückenmarksegmentes T6 auf.

Merke

Bei Symptomen einer Autonomen Dysreflexie besteht die wichtigste Handlung im Erkennen und sofortigen Ausschalten des triggernden Reizes, d. h. beispielsweise dem Abbruch der Manipulation bzw. der Untersuchung oder des Harnverhalts durch Leeren der Harnblase [26][4][5].

Bei anhaltender Symptomatik sollte eine rasche antihypertensive Therapie mit Nifedipin (5-10 mg oral), alternativ mit Urapidil $(6,25 \mathrm{mg}$ langsam i.v. ) erfolgen. Eine akute Senkung des Blutdrucks sollte ab der Lebensfähigkeit des Kindes unter CTG-Kontrollen erfolgen [6]. Das Krankheitsbild der Autonomen Dysreflexie ähnelt in wichtigen Symptomen der Präeklampsie, es stellen sich jedoch einige Unterschiede dar.

-Tab. 1 gibt einen Überblick über die Differenzialdiagnose einer Autonomen Dysreflexie und Präeklampsie.

\section{Hautveränderungen kontrollieren}

Aufgrund der Gewichtszunahme der gelähmten Schwangeren kann es zu Hautulzerationen kommen [37]. Deshalb sind regelmäßige Hautkontrollen und ggf. eine Hilfsmittelanpassung notwendig. Bei querschnittgelähmten Schwangeren sollten im 2. und 3. Trimenon mindestens zweimalige paraplegiologische und neurourologische Verlaufskontrollen in einem Querschnittgelähmten-Zentrum erfolgen. 


\section{Betreuung bei der Geburt}

Aufgrund des Risikos einer Autonomen Dysreflexie, ausgelöst durch Wehen bzw. Dehnungsreize beim Geburtsvorgang, benötigen alle Frauen mit einer Läsionshöhe oberhalb T6 eine möglichst frühzeitige, d. h. vor Einsetzen der Wehen wirkende Periduralanästhesie. Sie sollte als kontinuierliche patientenkontrollierte Analgesie (PCEA, patient-controlled epidural Analgesia) über ein Pumpensystem steuerbar sein, welches bis zum neurologischen Segment T10 reichen muss.

Bei der Gefahr einer Autonomen Dysreflexie soll eine verlängerte Kreißsaalüberwachung mit engmaschiger Vitalzeichenkontrolle bzw. ein erweitertes kontinuierliches Monitoring unter PDK bzw. PCEA für 48 Stunden erfolgen [15][28][11][36].

\section{Merke}

Die Querschnittlähmung einer schwangeren Frau stellt per se keine Indikation zur Durchführung einer primären Sectio dar [29]. Dennoch zeigen mehrere Studien, dass die Sectioquote bei Frauen mit QSL circa doppelt so hoch ist wie die Sectiorate allgemein [1]. Als Hauptindikation werden Frühgeburtlichkeit, ein fehlender Geburtsfortschritt sowie instabile maternale Beckenverhältnisse angegeben.

Die mangelnde Wahrnehmung von Kontraktionen führt in einigen Fällen zu ungeplanten Hausgeburten, unbeaufsichtigten Krankenhausgeburten oder Krankenhauseinweisungen in bereits fortgeschrittenem Geburtsvorgang [1]. Aus diesem Grund ist die Teilnahme an einer individuellen Geburtsvorbereitung, ggf. im häuslichen Umfeld der Schwangeren, empfehlenswert. Hierbei kann z. B. der Partner/die Partnerin ebenso wie die Schwangere die Palpation des Uterus zur Bestimmung von Frequenz und Stärke der Wehen erlernen.

\section{Geburtsverlauf}

- Die Eröffnungsperiode bei Frauen mit QSL verläuft prinzipiell ähnlich wie bei nicht querschnittgelähmten Frauen. In der Austreibungsperiode kann jedoch bei motorischer Lähmung die Kraft der willkürlich ansteuerbaren Bauch- und Beckenmuskulatur fehlen bzw. abgeschwächt sein. Während der Geburt sollte möglichst eine Eins-zu-eins-Betreuung durch die Hebamme in enger Absprache mit einem Facharzt für Frauenheilkunde und Geburtshilfe erfolgen. Auf die Zeichen der oben beschriebenen Autonomen Dysreflexie sowie auf ein regelmäßiges Blasen- und Darmmanagement sollte engmaschig geachtet werden. Die suffiziente Atmung der Gebärenden sollte beispielsweise durch Hochlagerung des Oberkörpers bei Paraplegikerinnen bzw. durch die flache Lagerung bei Tetraplegikerinnen gewährleistet werden [17].

- Aufgrund der teilweise eingeschränkten aktiven Mitwirkung der Frau in der Austreibungsperiode ist die Rate 
-Tab. 1 Differenzialdiagnose der autonomen Dysreflexie und Präeklampsie

\begin{tabular}{|c|c|c|}
\hline & Autonome Dysreflexie & Präeklampsie \\
\hline Lähmungshöhe & $\begin{array}{l}\text { meist oberhalb des } \\
\text { neurologischen Seg- } \\
\text { mentes T6, selten } \\
\text { darunter }\end{array}$ & $\begin{array}{l}\text { unabhängig von der } \\
\text { Lähmungshöhe }\end{array}$ \\
\hline Blutdruck & $\begin{array}{l}\text { anfallsweise extrem } \\
\text { hoch }\end{array}$ & $\begin{array}{l}\text { langsam kontinuierlich } \\
\text { steigend }\end{array}$ \\
\hline Herzfrequenz & $\begin{array}{l}\text { meist bradykard, in der } \\
\text { frühen Phase tachykard }\end{array}$ & meist normofrequent \\
\hline Proteinurie & nein & ja (>300 mg/24h) \\
\hline Klinik & $\begin{array}{l}\text { „Flushing”, Schwitzen, } \\
\text { Gänsehaut, pochende } \\
\text { Kopfschmerzen, gestei- } \\
\text { gerte Reflexe }\end{array}$ & $\begin{array}{l}\text { Ödeme, kontinuierli- } \\
\text { cher Kopfschmerzen, } \\
\text { Augenflimmern, Ober- } \\
\text { bauchschmerzen, } \\
\text { gesteigerte Reflexe }\end{array}$ \\
\hline $\begin{array}{l}\text { Serologische } \\
\text { Auffälligkeiten }\end{array}$ & untypisch & $\begin{array}{l}\text { Möglich z. B. bei gleich- } \\
\text { zeitigem HELLP-Syn- } \\
\text { drom (Harnsäure } \uparrow \text {, } \\
\text { Transaminasen } \uparrow, \\
\text { Thrombozyten } \downarrow \text {, } \\
\text { Haptoglobin } \downarrow \text { ) }\end{array}$ \\
\hline
\end{tabular}

an vaginaloperativen Geburten erhöht. Eine Dammverletzung und Episiotomie sollte aufgrund der möglicherweise erschwerten Nahtheilung vermieden werden. Entsprechend sind Geburten in Rückenlage auch für Frauen mit Querschnittlähmung ungünstig [21][41].

- Die Plazentarperiode unterscheidet sich nicht von Frauen ohne Querschnittlähmung. Die Frau wird, sofern kein Risiko einer Autonomen Dysreflexie vorliegt, postpartal wie gewohnt für zwei Stunden im Kreißsaal betreut. Bei höher gelähmten Frauen und dem erhöhten Risiko einer Autonomen Dysreflexie sollte eine kontinuierliche Beobachtung über 48 Stunden sichergestellt werden. Untersuchungen zeigen, dass auch für Frauen mit einer QSL ein positives Geburtserlebnis von besonderer Bedeutung ist [14].

\section{Interdisziplinäre Betreuung im Wochenbett}

Die Betreuung im Wochenbett sollte im Team von Gynäkologen, Paraplegiologen, Neuro-Urologen und Hebammen erfolgen. Aufgrund eines veränderten Tonus der Bauch- und Beckenmuskulatur kann der Fundus uteri tiefer palpiert werden als bei Frauen ohne Querschnittlähmung [37]. Bei fehlender bzw. eingeschränkter Schmerzsymptomatik wird häufig ein Lochialstau nicht oder spät erkannt, sodass hierauf besonderes geachtet werden sollte.

\section{Merke}

Eine Querschnittlähmung stellt keine Kontraindikation für das Stillen dar. Mütter mit QSL sollten vielmehr zum Stillen ermutigt werden.

Bei Läsionen unterhalb von T5 ist typischerweise ein problemloses Stillen möglich, es kann jedoch zu einer Triggerung der Spastik kommen. Bei einer Läsion oberhalb von T5, ist mit einer aufgehobenen bzw. eingeschränkten Sensorik der Mamillen und einer eventuell reduzierten Milchbildung zu rechnen. Auch hier sollten nochmals die gängigen Medikamente auf die Verträglichkeit in der Stillzeit überprüft werden. Bei zwingender Indikation zur medikamentösen Therapie sollte ein Abstillen erfolgen.

\section{FAZIT}

Zusammenfassend lässt sich konstatieren, dass die Betreuung einer Frau mit Querschnittlähmung in der Schwangerschaft und während der Geburt eine interdisziplinäre Herausforderung für Ärzte, Paraplegiologen und Hebammen darstellt. Die S2k-Leitlinie "Schwangerschaft und Geburt bei Frauen mit Querschnittlähmung“ soll hierbei die Zusammenarbeit unterstützen und helfen, Ängste zu überwinden und Hilfestellungen bei der Betreuung dieser Gruppe von Frauen geben. Es ist die Aufgabe der Frauenärzte, Paraplegiologen und Hebammen, querschnittgelähmte Frauen über Risiken und Prophylaxen aufzuklären, ihnen aber nicht den Mut für eine Schwangerschaft zu nehmen. In der fachübergreifenden Zusammenarbeit der Berufsgruppen besteht eine große Chance, den Frauen und ihren Familien trotz einiger Risiken ein positives Schwangerschafts-, Geburts- und Wochenbetterleben zu ermöglichen.

\section{Autorinnen / Autoren}

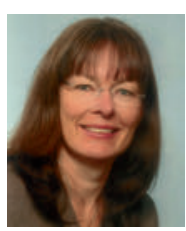

Prof. Dr. rer. medic. Ute Lange ist Hebamme und Professorin für Hebammenwissenschaft an der Hochschule für Gesundheit in Bochum.

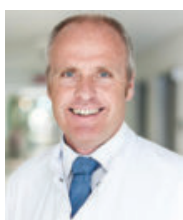

Prof. Dr. med. Markus Schmidt ist Facharzt für Gynäkologie und Geburtshilfe sowie Chefarzt der Klinik für Gynäkologie und Geburtshilfe der Sana Kliniken Duisburg.

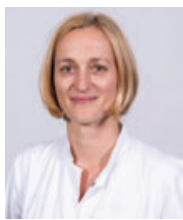

Dr. med. Ines Kurze ist Fachärztin für Urologie, Chefärztin, Querschnittgelähmten-Zentrum / Klinik für Paraplegiologie und Neuro-Urologie, Zentralklinik Bad Berka. 
hsg Bochum | Hochschule für Gesundheit

Studienbereich Hebammenwissenschaft

Prof. Dr. Ute Lange

Gesundheitscampus $6-8$

44801 Bochum

E-Mail: ute.lange@hs-gesundheit.de

\section{Literatur}

[1] American College of Obstetricians and Gynecologists (ACOG). ACOG committee opinion. Obstetric management of patients with spinal cord injuries. Number 275, September 2002. Committee on Obstetric Practice. Int J Gynecol Obstet 2002; 79(2): 189-191

[2] Arbeitsgemeinschaft der Wissenschaftlichen Medizinischen Fachgesellschaften e.V. (AWMF). AWMF-Leitlinie 179-002: Schwangerschaft, Geburt und Wochenbett bei Frauen mit Querschnittlähmung. Im Internet: https://www.awmf.org/upl oads/tx_szleitlinien/179-002I_S2k_Wochenbett-Schwanger schaft-Geburt-Querschnittlaehmung_2018-10_1.pdf; Stand: $09 / 2018$

[3] Arbeitsgemeinschaft der Wissenschaftlichen Medizinischen Fachgesellschaften e.V. (AWMF). AWMF- Leitlinie 179-004: Neurogene Darmfunktionsstörungen bei Querschnittlähmung. Im Internet: https://www.awmf.org/uploads/tx_szleitli nien/179-004I_S2k_Neurogene-Darmfunktionsstoerung-Que rschnittlaehmung_2019-10_1.pdf; Stand: 08 / 2019

[4] Arbeitsgemeinschaft der Wissenschaftlichen Medizinischen Fachgesellschaften e.V. (AWMF). AWMF-Leitlinie 179-001: Neuro-urologische Versorgung querschnittgelähmter Patienten. Im Internet: https://www.awmf.org/uploads/tx_szleitli nien/179-001I_S2k_Neurourologische_Versorgung_Quer schnittsgelaehmter_2019-10-verlaengert.pdf

[5] Arbeitsgemeinschaft der Wissenschaftlichen Medizinischen Fachgesellschaften e.V. (AWMF). AWMF-Leitlinie 030-070: Querschnittlähmung. Stand: 09/2012

[6] Arbeitsgemeinschaft der Wissenschaftlichen Medizinischen Fachgesellschaften e.V. (AWMF). AWMF-Leitlinie 015/018: Diagnostik und Therapie hypertensiver Schwangerschaftserkrankungen. https://www.awmf.org/uploads/tx_szleitlinien/0 15-018I_S2k_Diagnostik_Therapie_hypertensiver_Schwanger schaftserkrankungen_2019-07.pdf; Stand: 03 / 2019

[7] Bertschy S, Bostan C, Meyer T et al. Medical complications during pregnancy and childbirth in women with SCl in Switzerland. Spinal Cord 2016; 54(3): 183-187.

[8] Bertschy S, Pannek J, Meyer T. Decision making under uncertainty: Swiss providers' experiences in caring for women with spinal cord injury during pregnancy and childbirth - an expert interview study. BMC Pregnancy and Childbirth 2016; 16(1): 181

[9] Bertschy S. Maternity Care Services for Women with Disabilities during Pregnancy and Childbirth in Switzerland: Learning from the experiences of women with spinal cord injuries and their practitioners to optimise maternity care services [Dissertation]. Luzern, Schweiz: Universität Luzern 2017, DOI:10.5281 / zenodo.1209422.

[10] Bundesverband behinderter und chronisch kranker Eltern e. V. (bbe e. V.). Blochberger K. Bösemann T, Frevert U et al. Elternassistenz. Im Internet: http://www.behinderte-eltern.d e/Papoo_CMS/index.php?menuid=75; Stand: 2015 
[11] Burns R, Clark VA. Epidural anaesthesia for caesarean section in a patient with quadriplegia and autonomic hyperreflexia. Int J Obstet Anesth 2004; 13(2):120-123

[12] Camune BD. Challenges in the management of the pregnant woman with spinal cord injury. J Perinat Neonatal Nurs 2013; 27(3):225-231

[13] Cavalli P. Prevention of Neural Tube Defects and proper folate periconceptional supplementation. J Prenat Med 2008; 2 (4):40-41

[14] Cowley KC. Breastfeeding by women with tetraplegia: some evidence for optimism. Spinal Cord 2014; 52, 255; DOI:10.1038/sc.2013.167

[15] Dawood R, Altanis E, Ribes-Pastor P et al. Pregnancy and Spinal Cord Injury. The Obstetrician \& Gynaecologist 2014: 16 (2): 99-107

[16] Deutsche Gesellschaft für Ernährung e. V. (DGE). Referenzwerte für die Nährstoffzufuhr: Folat. Im Internet: http://ww w.dge.de/wissenschaft/referenzwerte/folat/; Stand: 07/ 2013

[17] Deutsches Netzwerk für Qualitätsentwicklung in der Pflege (DNQP) und Verbund Hebammenforschung, Hrsg: Expertinnenstandard „Förderung der physiologischen Geburt”. Entwicklung - Konsentierung - Implementierung. Schriftenreihe des Deutschen Netzwerks für Qualitätsentwicklung in der Pflege, Osnabrück; 2014

[18] Ghidini A, Healey A, Andreani M et al. Pregnancy and women with spinal cord injuries. Acta Obstet Gynecol Scand 2008; 87 (10):1006-1010

[19] Ghidini A, Simonson M. Pregnancy after spinal cord injury: a review of the literature. Top Spinal Cord Inj Rehabil 2011; 8 (3): $20-25$

[20] Gynecologists ACoOa: Clinical Management Guidelines for Obstetrician-Gynecologists. Prevention of deep vein thrombosis and pulmonary embolism. Obstet Gynecol 2007; 84 (110):429-440

[21] Hughes S], Short D], Usherwood MM et al. Management of the pregnant woman with spinal cord injuries. $\mathrm{Br} J$ Obstet Gynaecol 1991; 98(6):513-518

[22] lezzoni LI, Yu J, Wint AJ et al. Prevalence of current pregnancy among US women with and without chronic physical disabilities. Med Care 2013; 51(6):555-562

[23] Jackson A, Lindsey L, Klebine P. Reproductive health for women with spinal cord injury: pregnancy and delivery. SCI Nurs 2004; 21(2):88-91

[24] Jackson AB, Wadley V: A multicenter study of women's selfreported reproductive health after spinal cord injury. Arch Phys Med Rehabil 1999; 80(11):1420-1428

[25] Khunda A, Karmarkar R, Abtahi Ba et al. Pregnancy in women with Fowler's syndrome treated with sacral neuromodulation. Int Urogynecol J 2013; 24: 1201-1204

[26] Krassioukov A, Warburton DE, Teasell $R$ et a. A systematic review of the management of autonomic dysreflexia after spinal cord injury. Arch Phys Med Rehabil 2009; 90(4):682-695

[27] Kuczkowski KM. Labor analgesia for the parturient with spinal cord injury: what does an obstetrician need to know? Arch Gynecol Obstet 2006; 274(1):41-46
[28] Kuczkowski KM. Peripartum anaesthetic management of a parturient with spinal cord injury and autonomic hyperreflexia. Anaesthesia 2003; 58(8):823-824

[29] Le Liepvre H, Dinh A, Idiard-Chamois B et al. Pregnancy in spinal cord-injured women, a cohort study of 37 pregnancies in 25 women. Spinal Cord 2016; 55(2):167-171

[30] Leder D, Geng V. Ernährung und Verdauung: Physiologie und Pathophysiologie des Gastrointestinaltrakts. In: Zäch GA, Koch HG, Hrsg. Paraplegie. Ganzheitliche Rehabilitation. Basel: Karger; 2006: 157-162

[31] Mamopoulos A, Stavrakis T, Mavromatidis G et al. Active sacral neuromodulator during pregnancy: a unique case report. American Journal of Obstetrics and Gynecology 2014; 211(1): e4-e5

[32] Manfred-Sauer-Stiftung. Geng V, Obereisenbuchner ], Senft B et al. Ernährungsempfehlung für Querschnittgelähmte. Im Internet: https://www.der-querschnitt.de/wp-content/uplo ads/2014/12/B_Empfehlung-Ernährung-QS-1405.pdf Stand: 2014

[33] Morton C, Le JT, Shahbandar L et al. Pregnancy outcomes of women with physical disabilities: a matched cohort study. PM R 2013, 5(2):90-98

[34] Pannek J, Bertschy S. Mission impossible? Urological management of patients with spinal cord injury during pregnancy: a systematic review. Spinal Cord 2011; 49(10):1028-1032

[35] Roulette P, Castel-Lacanal E, Sanson S et al. Sacral neuromodulation and pregnancy: Results of a national survey carried out for the neuro-urology committee of the French Association of Urology (AFU). Neurourol Urodyn 2017; 37(2): 792-798; DOI: 10.1002 / nau.23349

[36] Sharpe EE, Arendt KW, Jacob AK et al. Anesthetic management of parturients with pre-existing paraplegia or tetraplegia: a case series. Int J Obstet Anesth 2015; 24(1):77-84

[37] Signore C, Spong CY, Krotoski D et al. Pregnancy in women with physical disabilities. Obstet Gynecol 2011; 117(4):935-947

[38] Sterling L, Keunen J, Wigdor E et al. Pregnancy outcomes in women with spinal cord lesions. Journal of obstetrics and gynaecology Canada (JOGC) 2013; 35(1):39-43

[39] Tebbet M, Kennedy P. The experience of childbirth for women with spinal cord injuries: an interpretative phenomenology analysis study. Disability and Rehabilitation 2012; 34(9-10): 762-769

[40] Wiseman O], Hombergh UVD, Koldewijn EL et al. Sacral neuromodulation and pregnancy. J Urol 2002; 167: 165-168

[41] Zäch A, Koch HG. Atmung bei Querschnittlähmung. In: Zäch A, Koch HG, Hrsg. Paraplegie. Ganzheitliche Rehabilitation. Basel: Karger; 2006: 126, 128-130

\section{Bibliografie}

DOI https://doi.org/10.1055/a-1084-5097

Die Hebamme 2020; 33: 24-30

(c) Georg Thieme Verlag KG Stuttgart · New York ISSN 0932-8122 